Gutiérrez Sánchez, M. y Vidal Valenzuela, S. (2020). La Escala Observacional de Memoria Operativa (EOMO) como instrumento e icaz en la prevención y detección de di icultades de aprendizaje. Revista de Investigación Educativa, 38(1), 53-69.

DOI: http://dx.doi.org/10.6018/rie.313271

\title{
La Escala Observacional de Memoria Operativa (EOMO) como instrumento eficaz en la prevención y detección de dificultades de aprendizaje
}

\section{The Observational Scale of Working Memory (EOMO) as an effective instrument in the prevention and detection of learning difficulties}

\author{
Marta Gutiérrez Sánchez* y Sergio Vidal Valenzuela** \\ *Departamento de Teoría e Historia de la Educación. Facultad de Educación. Universidad de Murcia \\ **Equipo de Orientación Educativa y Psicopedagógica Murcia 2. Consejería de Educación y Cultura de la Región de \\ Murcia
}

\begin{abstract}
Resumen
El presente trabajo propone una optimización de los procesos de prevención y detección de dificultades de aprendizaje en el contexto educativo mediante el estudio de la relación que la Escala Observacional de Memoria Operativa (EOMO) muestra con medidas de la eficacia lectora, comprensión lectora, rendimiento matemático y rendimiento académico. Para ello, se estudió una muestra incidental de 636 alumnos de educación primaria, pertenecientes a centros educativos de la comarca de Cartagena. Los datos mostraron relaciones significativas de la EOMO con las diferentes variables medidas, y en el sentido esperado. Los resultados indicaron que la EOMO puede ser una herramienta de filtrado o screening que puede delimitar la muestra de alumnado que ha de ser valorada con mayor detalle, a fin de prevenir o detectar dificultades de aprendizaje.

Palabras clave: educación primaria; alumnado; dificultad de aprendizaje; rendimiento académico; diagnóstico.
\end{abstract}

Correspondencia: Marta Gutiérrez Sánchez, martags@um.es. Departamento de Teoría e Historia de la Educación, Facultad de Educación, Universidad de Murcia, Campus Universitario de Espinardo, 30100, Murcia (España) 


\begin{abstract}
The present study aims to improve the effectiveness of the process of preventing and detecting learning disabilities by studying the relationship between the Observational Scale of Working Memory (EOMO) and effective reading, reading comprehension, mathematical achievement, and academic achievement. The incidental sample consisted of 636 students from Primary Education, belonging to different schools in Cartagena. As expected, the results showed significant relationships between EOMO and all the variables measured. These findings showed that EOMO can be used as a screening tool to limit the sample of students that should be assessed in detail so as to prevent and detect learning disabilities.

Keywords: primary education; pupil; learning difficulty; academic achievement; diagnosis.
\end{abstract}

\title{
Introducción
}

La prevención de las dificultades de aprendizaje (en adelante, DA) sigue ocupando un lugar privilegiado en la normativa que regula nuestro actual sistema educativo. Así, la Ley Orgánica 8/2013, de 9 de diciembre, para la mejora de la calidad educativa (LOMCE) establece en su artículo 79bis que "corresponde a las administraciones educativas adoptar las medidas necesarias para identificar al alumnado con dificultades específicas de aprendizaje y valorar de forma temprana sus necesidades"(p.37). Desde el ámbito regional, y tributario del artículo anteriormente planteado, la Comunidad Autónoma de la Región de Murcia establece en el Decreto 359/2009, de 30 de octubre, por el que se establece y regula la respuesta educativa a la diversidad del alumnado, la necesidad de "mejorar la detección y prevención de las dificultades de aprendizaje anticipándose a las mismas y evitando situaciones de abandono, fracaso o inadaptación escolar, personal o social" (p. 57638).

Partiendo de estas premisas, desde 2012, la Consejería de Educación de la Región de Murcia establece como prescriptivos los planes de prevención y detección de DA en los centros escolares dependientes de la misma, legislando este hecho en la Resolución de 17 de diciembre de 2012, de la Dirección General de Planificación y Ordenación Educativa. A pesar de este planteamiento, la Consejería no define protocolos para el desarrollo de tales planes, siendo el criterio del centro escolar, apoyado y legitimado por el equipo de orientación educativa y psicopedagógica adscrito al mismo, el que determina cómo se desarrollan estos planes. En este contexto, en un sector educativo de Cartagena se viene desarrollando, a partir del trabajo de Vidal (2012), un plan de prevención de DA en educación primaria que valora variables como la eficacia y la comprensión lectoras y el rendimiento matemático. Dicho plan ha resultado efectivo para la prevención-detección de DA, pese a la elevada inversión de tiempo y recursos que supone la valoración y el análisis de resultados del alumnado de cada uno de los niveles educativos a los que se destina este plan.

En este sentido, y con el objetivo de optimar el proceso de prevención-detección de DA, el uso de instrumentos psicométricamente validados, así como ágiles en su corrección, que tuvieran correlación con las habilidades instrumentales medidas (eficacia 
lectora, comprensión lectora y rendimiento matemático) podría servir de filtrado para determinar a aquel alumnado destinatario de una evaluación más amplia.

En esta línea, la memoria operativa (en adelante, MO) ha mostrado, en el ámbito experimental, relaciones significativas con la lectura y las matemáticas, así como con el rendimiento académico. Sin embargo, la medición de este constructo cognitivo siempre ha requerido de la administración individual de pruebas o administraciones informatizadas, que precisan de mucho tiempo para su aplicación y corrección. Sin embargo, la Escala Observacional de Memoria Operativa (EOMO) (Gómez et al., 2012) se presenta como una prueba con índices elevados de fiabilidad y validez, para medir de forma ágil y eficaz la MO. Se completa de manera rápida por parte del profesorado, y refleja la frecuencia de aparición de correlatos conductuales de las dificultades en MO. Cuanto mayor es la puntuación en la EOMO, mayores son las dificultades en MO. En el caso de que correlacionara con medidas de eficacia y comprensión lectora, y rendimiento matemático, podría constituir un indicador previo y fiable de posibles DA. De ser así, la utilización de esta escala supondría una gestión eficiente en el desarrollo de los planes de prevención de DA en educación primaria.

En los últimos treinta años se han venido desarrollando trabajos que permiten estudiar la relación de la MO con el aprendizaje escolar. La concepción de la MO ha ido evolucionando desde la visión de un mecanismo de almacenamiento temporal a modelos complejos y multi-componenciales, donde la relevancia radica en el papel activo y funcional de la MO en el procesamiento de la información (Gutiérrez, GarcíaMadruga, Elosúa, Luque y Gárate, 2002). En este sentido, es el modelo de Baddeley y Hitch (Baddeley, 2003; Baddeley y Hitch, 1994) el que ha venido considerándose en la investigación científica. En su propuesta inicial, considera que la MO está constituida por, al menos, cuatro subsistemas (ejecutivo central, buffer episódico, bucle fonológico y agenda viso espacial) organizados jerárquicamente y que actúan en estrecha colaboración para la manipulación y almacenamiento temporal de la información a fin de poder ejecutar tareas mentales de complejidad variable, como son la comprensión del lenguaje o el propio rendimiento académico.

La MO se relaciona íntimamente con el desarrollo cognitivo y el aprendizaje, así como con el rendimiento académico. Más específicamente, para los fines de este trabajo, la MO muestra también una relación significativa con el desarrollo de la lectura, la ejecución de la comprensión lectora y las matemáticas.

En cuanto a la comprensión lectora, son numerosos los estudios que han demostrado su relación con la MO. Así, Durán et al. (2016) mostraron que esta variable es un potente predictor de los resultados en conciencia fonológica, un requisito previo de la lectura; Gómez-Veiga, Vila, García-Madruga, Contreras y Elosúa (2013), señalaron que las puntuaciones en $\mathrm{MO}$ correlacionaban positivamente, junto con la inteligencia fluida, con las puntuaciones en comprensión lectora; y Esquivel, Martínez, Córdoba y Reyes (2016) y Guzmán, Véliz y Reyes (2017) evidenciaron que el papel de la MO en la comprensión sigue presente a lo largo de la vida académica.

Por otro lado, es conocida la influencia diferencial que las diferentes estructuras de la MO tienen a lo largo del proceso de aprendizaje matemático, así como en los procesos matemáticos propiamente dichos. Los estudios evidencian un papel relevante de la agenda viso espacial en la adquisición de los primeros conceptos matemáticos, 
siendo el bucle fonológico el responsable de aplicar los conceptos aprendidos (Raghubar, Barnes y Hecht, 2010). La agenda viso espacial también juega un papel fundamental en la escritura de números y la estimación de magnitudes, erigiéndose como crucial para la resolución de problemas numéricos y textuales (Ashkenazi, Rosenberg-Lee, Metcalfe, Swigart y Menon, 2013). Si bien la agenda visoespacial se erige como un componente clave en la resolución de problemas, debemos también señalar estudios donde, sin restar relevancia a este componente, es el ejecutivo central el que juega el papel más significativo (Hubber, Gilmore y Cragg, 2014; Friso-Van den Bos, Van der Ven, Kroesbergen y Van Luit, 2013; Kolkman, Hoijtink, Kroesbergen y Leseman, 2013). Kroesbergen, Noordende y Kolkman (2014) analizaron la relación entre MO y los inicios de la aritmética, estudiando la posible relación entre el entrenamiento en $\mathrm{MO}$ y los posibles efectos que se pudieran derivar del mismo en el dominio específico de las habilidades numéricas tempranas. En niños de cinco años, los resultados comprobaron que el entrenamiento en MO no sólo mejoraba la misma, sino que también mejoraba la ejecución matemática.

También se ha demostrado que la MO presenta un funcionamiento diferencial en niños con DA, siendo las dificultades en lectura y en matemáticas los ámbitos más estudiados para conocer la relación de éstas con la MO. En el estudio de Gathercole et al. (2016) se evidenció que tres cuartas partes del alumnado con bajo rendimiento en lectura y matemáticas mostraban bajas puntuaciones en al menos una de las medidas de MO utilizadas, apuntando la importancia de considerar la carga en MO en los procesos de enseñanza-aprendizaje que se desarrollan en el aula.

Los datos con respecto a $\mathrm{MO}$ y las dificultades en lectura parecen mostrar fallos en la mayoría de componentes del modelo de $\mathrm{MO}$ anteriormente planteado, como en el funcionamiento ejecutivo en general (Kudo, Lussier y Swanson, 2015). El trabajo de Moura, Simões y Pereira (2015), demostró que, en niños con dislexia, el bucle fonológico y el ejecutivo central son los dos componentes que peor ejecución mostraban en alumnado con dificultades en lectura, siendo la ejecución de estos dos componentes un predictor significativo de la habilidad lectora. Fischbach, Könen, Rietz y Hasselhorn (2014) realizaron un estudio longitudinal comparando el funcionamiento de la $\mathrm{MO}$ del alumnado con dificultades en lectura y normo lectores. Como en el trabajo anterior, se mostraba un peor funcionamiento del bucle fonológico y del ejecutivo central.

La evidencia científica muestra también relaciones entre las dificultades en matemáticas y déficits en MO. Existe una relación estrecha entre bajas puntuaciones en tareas de MO y dificultades de cálculo. Klesczewski et al. (2018) llevaron a cabo un estudio evolutivo, de tercero a quinto grado, de la $\mathrm{MO}$ en el alumnado con y sin dificultades en matemáticas. Sus resultados demostraron que las puntuaciones en MO (bucle fonológico, ejecutivo central y agenda visoespacial) son mayores en el alumnado sin dificultades en matemáticas. Evidenciaron también que el desarrollo de los componentes de la $\mathrm{MO}$ en el alumnado con dificultades en matemáticas mostraba paralelismos con el grupo control en el ejecutivo central y el bucle fonológico, no siendo así con respecto a la agenda visoespacial. Los datos de McDonald y Berg (2017) mostraron datos similares, al proponer que los fallos en $\mathrm{MO}$ en el alumnado con dificultades en matemáticas pueden estar reflejando más un retraso en el desarrollo que un déficit de este componente cognitivo. 
También se ha demostrado que la MO es uno de los factores determinantes del rendimiento académico (García-Madruga y Fernández, 2008; Blankenship, O’Neill, Ross y Bell, 2015). El papel de la MO en el rendimiento académico se sustancia por el hecho de que el aprendizaje académico requiere del manejo de representaciones mentales de la información. Así, Alloway, Alloway y Wootan (2014) en un estudio longitudinal con niños en educación infantil cuyo objetivo era estudiar el valor predictivo de factores cognitivos, entre los que se encontraba la $\mathrm{MO}$, demostraron que, dos años después de haber evaluado los diferentes factores, la MO, junto con la conciencia fonológica, se erigían como los mejores predictores del rendimiento académico. Es reseñable que estas dos variables superaban en poder predictivo incluso a factores como el estatus socioeconómico. Similares son los resultados obtenidos por Maehler y Schuchardt (2016) al demostrar que $\mathrm{MO}$ es una variable que muestra déficits en aquel alumnado con un rendimiento escolar bajo, independientemente de la inteligencia. También destacar el trabajo de Gutiérrez-Martínez y Ramos (2014) quienes indican que la MO es disociable de la capacidad cognitiva.

De acuerdo a la literatura científica que ampara la relación entre MO y DA, y desde la pretensión de mejorar la intervención educativa en DA, los objetivos que se plantean con este trabajo son los siguientes:

1. Analizar la relación entre las puntuaciones de la EOMO y la eficacia lectora, la comprensión lectora, el rendimiento matemático y el rendimiento académico; a fin de considerar a esta escala como un filtro para determinar a aquellos alumnos y alumnas a valorar en los planes de prevención y detección de DA.

2. Comprobar la eficacia y fiabilidad de la EOMO para predecir los resultados de eficacia y comprensión lectoras, rendimiento matemático y rendimiento académico.

Por tanto, las hipótesis del estudio son dos. La primera plantea que las puntuaciones en la EOMO correlacionan en sentido negativo con todas las variables medidas, en todos los cursos escolares. La EOMO mide la frecuencia de aparición de patrones conductuales compatibles con dificultades en la MO. De esta forma, cuanto mayor es la puntuación otorgada por el tutor, mayores son las dificultades en MO. En este sentido, la EOMO se relaciona de forma inversa con el resto de variables medidas. Así, cuanto mayores sean las puntuaciones en la Escala EOMO, menor será la competencia en eficacia lectora, comprensión lectora y cálculo, así como menor será el rendimiento académico. La segunda hipótesis propone que la EOMO es un predictor fiable de las diferentes competencias valoradas, así como del rendimiento académico.

\section{Diseño y método}

\section{Instrumentos}

Los instrumentos utilizados en esta investigación son los que utilizan los Equipos de Orientación Educativa y Psicopedagógica dependientes de la Consejería de Educación de la Región de Murcia para diagnosticar dificultades de aprendizaje. 
Si bien fueron desarrollados hace unos años se siguen utilizando porque muestran patrones de eficacia y validez para el diagnóstico en educación primaria. Para valorar el rendimiento en eficacia lectora y comprensión lectora se utilizaron dos pruebas: el Test Colectivo de Eficacia Lectora (TECLE) y el Test de Evaluación de Comprensión Lectora (ACL).

El TECLE (Marín y Carrillo, 1999) es una prueba de aplicación colectiva. Se compone de 64 ítems, en los que se debe elegir, de entre 4 opciones, la palabra que completa la frase. Las frases se presentan en orden de dificultad creciente, controlando la complejidad sintáctica, semántica y ortográfica de cada frase. Existen distractores fonológicos, ortográficos y semánticos. Contiene dos ítems de ensayo y la prueba es cronometrada, con una duración de cinco minutos en educación primaria. La puntuación global se obtiene de restar al total de aciertos el número de errores partido por tres, a fin de eliminar el efecto del azar, siendo el máximo de puntuación posible 64 puntos.

El ACL (Catalá, Catalá, Molina y Monclús, 2001) es un conjunto de 6 pruebas, una para cada uno de los seis cursos de educación primaria, que se dirige a valorar la comprensión lectora de forma amplia, con textos diversos, y con temáticas relativas a las diferentes materias curriculares. Estas pruebas recogen información sobre cuatro dimensiones relevantes de la comprensión lectora: la literal, la reorganizativa, la inferencial y la crítica. La comprensión literal se concentra en las ideas e informaciones que están explícitas en el texto y supone reconocer la información que el texto presenta de manera literal. La reorganización supone que el alumnado analice, sintetice y/o organice las ideas explícitamente manifiestas en el texto. La comprensión inferencial es la que se ejecuta cuando el alumno/a es capaz de usar simultáneamente la información explicitada en el texto por un lado y, por otro, los conocimientos previos que posee, para la elaboración de hipótesis. La comprensión crítica supone emitir respuestas que indiquen que el alumno/a ha hecho un juicio evaluativo, confrontando las ideas expuestas en el texto con un criterio interno (personal) o externo (el profesor, otras fuentes, etc.). Para los fines de este trabajo se consideró el porcentaje de aciertos de cada alumno/a en el global de la prueba, puesto que los textos son diferentes para cada nivel educativo, presentando un número de ítems variable según el nivel.

Para valorar el rendimiento matemático se administró la Batería de Aptitudes Diferenciales y Generales (BADYG) (Yuste, Martínez y Galve, 2011) la cual consta de 7 pruebas comunes a todos los niveles; aunque para este trabajo se administraron dos sub-pruebas: la sub-prueba de Cálculo y la de Series Numéricas. La primera se administra desde primero a cuarto, mientras que la segunda se administra en los niveles de quinto y sexto de primaria. La prueba de Cálculo evalúa la rapidez y seguridad para realizar sencillos cálculos numéricos de sumas y restas. La prueba de Series Numéricas consiste en la presentación de series de números, tras las que se presentan varias opciones de respuesta de entre las cuales el alumno/a ha de inferir, mediante la elaboración de cálculos sencillos, cuál es el número que sigue, y elegirlo de entre las diferentes alternativas de respuesta. En ambas pruebas, la puntuación total consiste en restarle al total de aciertos los errores cometidos. 


\section{Población y Muestra}

La población objeto de estudio la conforman alumnos y alumnas de todos los niveles de la etapa de Educación Primaria. Los participantes fueron seleccionados mediante un muestro no probabilístico incidental, quedando la muestra configurada en 636 participantes, pertenecientes a dos centros escolares públicos de la comarca de Cartagena de similar contexto socioeconómico. En concreto, el alumnado de estos centros procede de familias con estudios secundarios en su mayoría y una ocupación en actividades agrícolas, industriales y de hostelería y restauración. Los estudiantes pertenecían a diferentes niveles educativos (96 alumnos en primero de primaria, 124 alumnos en segundo de primaria, 115 alumnos en tercero de primaria, 91 alumnos en cuarto de primaria, 123 alumnos en quinto de primaria y 87 alumnos en sexto de primaria). El rango de edad de la muestra fue de 5 hasta los 12 años. Dado que el enfoque de este estudio es preventivo, para el análisis de los datos se descartó de la muestra al alumnado que presentaba informe psicopedagógico de necesidades específicas de apoyo educativo.

\section{Procedimiento de recogida y análisis de datos}

Para la administración de las diferentes pruebas se contó con la aprobación de los equipos directivos de los centros y con la colaboración de los orientadores responsables de los mismos. Esta administración de pruebas se llevó a cabo en el tercer trimestre del curso escolar 2015-2016. Se administró en primer lugar el TECLE. Posteriormente se administraron Cálculo (o Series Numéricas) y en último lugar se completaba el ACL. Se instruyó a los tutores sobre la administración de las pruebas. El alumnado fue dispuesto a modo de examen, indicándoles que lo que iban a realizar no tenía influencia en los resultados académicos.

Posteriormente se facilitó a los tutores de educación primaria la EOMO, que cumplimentaron para cada alumno/a.

Se recopilaron las notas finales de todo el alumnado en las siguientes materias: Lengua Castellana y Literatura, Matemáticas, Ciencias Naturales y Ciencias Sociales.

\section{Resultados}

Los datos de este estudio se analizaron con el programa estadístico SPSS v.29 (IBM SPSS Statistics for Windows, NY). En la Tabla 1, y con carácter descriptivo, se muestran la media y la desviación típica de todas las variables estudiadas: la nota final en cada una de las áreas consideradas, la nota media, y las puntuaciones obtenidas en la EOMO, el ACL, el TECLE y en las pruebas de Cálculo (de 1o a $4^{\circ}$ ) y Series Numéricas $\left(5^{\circ}\right.$ y $\left.6^{\circ}\right)$. Los estadísticos descriptivos se muestran agrupados por nivel educativo. Se aprecia un patrón ascendente en los resultados del TECLE a medida que se avanza en la etapa educativa. Igualmente se muestra un ascenso en las puntuaciones en las pruebas de rendimiento matemático. Sin embargo, en los resultados del ACL se percibe un decremento en la ejecución hacia cursos superiores. 
Tabla 1

Estadísticos descriptivos (media aritmética y desviación estándar entre paréntesis) del rendimiento académico, la comprensión lectora, la eficacia lectora y el rendimiento matemático. Organizado por cursos

\begin{tabular}{lcccccc}
\hline & $\mathbf{1}^{\mathbf{0}}$ & $\mathbf{2}^{\mathbf{0}}$ & $\mathbf{3}^{\mathbf{0}}$ & $\mathbf{4}^{\mathbf{0}}$ & $\mathbf{5}^{\mathbf{0}}$ & $\mathbf{6}^{\mathbf{0}}$ \\
\hline \multirow{2}{*}{ Lengua Castellana } & 8.56 & 7.87 & 6.90 & 7.09 & 6.94 & 6.74 \\
& $(1.33)$ & $(1.65)$ & $(1.65)$ & $(1.70)$ & $(1.56)$ & $(1.86)$ \\
Matemáticas & 8.10 & 7.90 & 6.48 & 6.93 & 6.59 & 6.24 \\
& $(1.44)$ & $(1.82)$ & $(2.06)$ & $(2.02)$ & $(1.64)$ & $(1.97)$ \\
Ciencias Naturales & 8.96 & 8.52 & 7.12 & 7.62 & 7.17 & 7.50 \\
Ciencias Sociales & $(.74)$ & $(1.47)$ & $(1.63)$ & $(1.61)$ & $(1.17)$ & $(1.86)$ \\
& 9.13 & 8.48 & 6.98 & 6.91 & 6.86 & 8.26 \\
Nota Media & $(.76)$ & $(1.45)$ & $(1.57)$ & $(1.66)$ & $(1.47)$ & $(1.67)$ \\
\multirow{2}{*}{ ACL (\% de Aciertos) } & 8.69 & 8.19 & 6.87 & 7.13 & 6.89 & 7.18 \\
& $(.91)$ & $(1.53)$ & $(1.65)$ & $(1.65)$ & $(1.33)$ & $(1.72)$ \\
TECLE & 52.83 & 66.47 & 47.24 & 57.53 & 48.34 & 44.84 \\
& $(25.58)$ & $(23.16)$ & $(22.8)$ & $(19.33)$ & $(16.93)$ & $(17.95)$ \\
Rendimiento Matemático & 13.79 & 22.05 & 27.43 & 35.05 & 41.35 & 46.50 \\
& $(7.75)$ & $(9.19)$ & $(9.56)$ & $(10.99)$ & $(10.57)$ & $(11.57)$ \\
& 11.23 & 14.42 & 15.50 & 19.16 & 18.90 & 20.17 \\
& $(5.12)$ & $(5.1)$ & $(6.28)$ & $(4.81)$ & $(5.44)$ & $(6.04)$ \\
\hline
\end{tabular}

En la Tabla 2 se muestran las correlaciones entre las diferentes pruebas y la EOMO. Como se puede apreciar, y tal como predecíamos en nuestras hipótesis de partida, todas las correlaciones muestran significación estadística, excepto la relación entre la eficacia lectora y la EOMO en sexto de primaria. Aunque significativa, también debemos señalar la baja correlación entre la EOMO y el rendimiento matemático en tercero de primaria. En general, las correlaciones van en el sentido esperado: cuanto mayor es la puntuación en la $\mathrm{EOMO}$, y por tanto se presentan más dificultades en $\mathrm{MO}$, menores son las puntuaciones en comprensión lectora, eficacia lectora y rendimiento matemático.

Tabla 2

Correlaciones de las pruebas ACL, TECLE y Cálculo con la EOMO. Organizado por cursos

\begin{tabular}{lcccccc}
\hline & $\mathbf{1}^{\mathbf{0}}$ & $\mathbf{2}^{\mathbf{0}}$ & $\mathbf{3}^{\mathbf{0}}$ & $\mathbf{4}^{\mathbf{0}}$ & $\mathbf{5}^{\mathbf{0}}$ & $\mathbf{6}^{\mathbf{0}}$ \\
\hline ACL & $-.556^{* *}$ & $-.670^{* *}$ & $-.485^{* *}$ & $-.682^{* *}$ & $-.384^{* *}$ & $-.401^{* *}$ \\
TECLE & $-.592^{* *}$ & $-.584^{* *}$ & $-.494^{* *}$ & $-.368^{* *}$ & $-.388^{* *}$ & -.157 \\
RENDIMIENTO MATEMÁTICO & $-.359^{* *}$ & $-.413^{* *}$ & $-.183^{*}$ & $-.589^{* *}$ & $-.349^{* *}$ & $-.514^{* *}$ \\
\hline
\end{tabular}

Nota: ${ }^{*}: \mathrm{p}<.05 ;{ }^{* *}: \mathrm{p}<.01 ;$ contraste unilateral) 
En la Tabla 3, y de acuerdo a la codificación de los alumnos/as en función de la puntuación obtenida en la EOMO, se muestran los resultados en las diferentes pruebas. Como se puede comprobar, la tendencia general es la esperada: cuanto mayor es la puntuación en la EOMO, las puntuaciones en las diferentes variables medidas tienden a disminuir. Con relación al TECLE, los grupos de EOMO alto muestran medias que se alejan una desviación típica de la media general, lo que los sitúa en el punto de corte para considerar dificultades en lectura. En sexto de primaria, la prueba TECLE no muestra la tendencia esperada (ver tabla 2). Parece que la demanda cognitiva para dar cuenta de esta prueba no es exigente a partir de ciertos niveles educativos. Estos datos pueden ser compatibles con el trabajo de Swanson (2003), donde se evidenciaba que el rendimiento de $\mathrm{MO}$ era menor en niños con dificultades de lectura, pero se hacía manifiesto en tareas que requerían de un procesamiento exigente.

Con respecto al ACL, los grupos de EOMO alto mostraban un rendimiento medio equivalente a niveles bajos ( $2^{\circ}$ curso) y muy bajos $\left(3^{\circ}, 4^{\circ}\right.$ y $6^{\circ}$ cursos), atendiendo a los baremos de la prueba.

El rendimiento matemático en los grupos de EOMO alto no superaba el percentil 25 de acuerdo a los baremos de la prueba usada (percentil 25 para $2^{\mathrm{o}}$ curso, percentil 19 para $3^{\circ}$, percentil 23 para $4^{\circ}$ y percentil 6 para $6^{\circ}$ )

Tabla 3

Resultados de las diferentes pruebas por puntuaciones. Los datos representan la media, y entre paréntesis la desviación típica. Organizado por cursos

\begin{tabular}{|c|c|c|c|c|c|c|c|}
\hline & & $1^{0}$ & $2^{o}$ & $3^{0}$ & $4^{0}$ & $5^{0}$ & $6^{0}$ \\
\hline \multirow{9}{*}{ TECLE } & \multirow{3}{*}{$\begin{array}{l}\text { EOMO } \\
\text { Bajo }\end{array}$} & $\mathrm{n}=89$ & $\mathrm{n}=88$ & $\mathrm{n}=81$ & $\mathrm{n}=79$ & $\mathrm{n}=112$ & $\mathrm{n}=78$ \\
\hline & & 14.81 & 24.92 & 30.06 & 36.05 & 41.77 & 46.11 \\
\hline & & $(7.42)$ & $(8.04)$ & $(8.55)$ & (10.19) & (10.72) & (11.78) \\
\hline & \multirow{3}{*}{$\begin{array}{l}\text { EOMO } \\
\text { Medio }\end{array}$} & $\mathrm{n}=7$ & $n=30$ & $n=23$ & $\mathrm{n}=8$ & $\mathrm{n}=11$ & $n=6$ \\
\hline & & 10.08 & 14.08 & 22.69 & 32.22 & 37.95 & 53.16 \\
\hline & & (3.09) & $(8.57)$ & (10.21) & (15.26) & (14.75) & (8.66) \\
\hline & \multirow{3}{*}{$\begin{array}{l}\text { EOMO } \\
\text { Alto }\end{array}$} & \multirow{3}{*}{-- } & $\mathrm{n}=6$ & $\mathrm{n}=11$ & $\mathrm{n}=4$ & & $\mathrm{n}=3$ \\
\hline & & & 12.11 & 18.05 & 19.16 & -- & 48.33 \\
\hline & & & $(4.23)$ & $(5.12)$ & $(9.04)$ & & $(7.32)$ \\
\hline \multirow{6}{*}{ ACL } & EOMO & 56.15 & 76.23 & 54.53 & 60.80 & 49.48 & 46.15 \\
\hline & Bajo & (25.32) & (17.83) & (20.82) & (17.54) & (16.89) & (17.79) \\
\hline & EOMO & 27.08 & 44.16 & 31.63 & 35.57 & 39.18 & 33.33 \\
\hline & Medio & $(4.98)$ & (16.72) & (18.01) & $(8.45)$ & (14.75) & $(6.41)$ \\
\hline & EOMO & \multirow{2}{*}{--} & 34.72 & 26 & 26.71 & \multirow{2}{*}{--} & 16.66 \\
\hline & Alto & & (13.08) & (15.77) & (16.49) & & (11.32) \\
\hline
\end{tabular}




\begin{tabular}{clcccccc}
\hline & & $\mathbf{1}^{\mathbf{0}}$ & $\mathbf{2}^{\mathbf{0}}$ & $\mathbf{3}^{\mathbf{0}}$ & $\mathbf{4}^{\mathbf{0}}$ & $\mathbf{5}^{\mathbf{0}}$ & $\mathbf{6}^{\mathbf{0}}$ \\
\hline \multirow{5}{*}{$\begin{array}{c}\text { Rendimiento } \\
\text { Matemático }\end{array}$} & EOMO & 11 & 15.73 & 16.48 & 20.10 & 19.21 & 20.51 \\
& Bajo & $(4.79)$ & $(3.48)$ & $(6.19)$ & $(3.41)$ & $(5.56)$ & $(5.76)$ \\
\cline { 2 - 8 } & EOMO & 8.25 & 11.47 & 11.18 & 13 & 16.43 & 19 \\
& Medio & $(6.25)$ & $(6.63)$ & $(5.94)$ & $(1.78)$ & $(3.67)$ & $(3.11)$ \\
\cline { 2 - 8 } Nota Media & EOMO & -- & 10 & 8.21 & 11 & & 5 \\
& Alto & & $(8.19)$ & $(4.09)$ & $(6.70)$ & - & $(5.40)$ \\
\hline & EOMO & 8.82 & 8.94 & 7.58 & 7.41 & 6.99 & 7.33 \\
& Eajo & $(0.75)$ & $(0.91)$ & $(1.31)$ & $(1.53)$ & $(1.30)$ & $(1.68)$ \\
\cline { 2 - 8 } & EOMO & 7.18 & 6.50 & 5.50 & 5.25 & 6.07 & 5.62 \\
& EOdio & $(1.07)$ & $(1.16)$ & $(0.94)$ & $(0.80)$ & $(1.26)$ & $(0.43)$ \\
\hline & EOMO & -- & 6.33 & 4.50 & 4.75 & & 4.25 \\
& Alto & & $(0.97)$ & $(0.72)$ & $(0.28)$ & - & $(0.35)$ \\
\hline
\end{tabular}

Nota: El doble guion significa que no había niños con una puntuación alta en la EOMO en los cursos de primero y quinto.

Igualmente, como puede apreciarse en la Tabla 4, la EOMO muestra correlaciones negativas significativas con el rendimiento académico, confirmándose la hipótesis de partida sobre la relación entre la EOMO y las distintas variables estudiadas.

Tabla 4

Correlaciones entre la EOMO y el rendimiento académico. Organizado por cursos

\begin{tabular}{lcccccc}
\hline & $\mathbf{1}^{\mathbf{0}}$ & $\mathbf{2}^{\mathbf{0}}$ & $\mathbf{3}^{\mathbf{0}}$ & $\mathbf{4}^{\mathbf{0}}$ & $\mathbf{5}^{\mathbf{0}}$ & $\mathbf{6}^{\mathbf{0}}$ \\
\hline Lengua Castellana & $-.420^{* *}$ & $-.747^{* *}$ & $-.618^{* *}$ & $-.692^{* *}$ & $-.548^{* *}$ & $-.551^{* *}$ \\
Matemáticas & $-.660^{* *}$ & $-.687^{* *}$ & $-.701^{* *}$ & $-.681^{* *}$ & $-.606^{* *}$ & $-.718^{* *}$ \\
Ciencias Naturales & $-.658^{* *}$ & $-.790^{* *}$ & $-.724^{* *}$ & $-.670^{* *}$ & $-.470^{* *}$ & $-.554^{* *}$ \\
Ciencias Sociales & $-.667^{* *}$ & $-.773^{* *}$ & $-.734^{* *}$ & $-.630^{* *}$ & $-.446^{* *}$ & $-.554^{* *}$ \\
Nota Media & $-.692^{* *}$ & $-.781^{* *}$ & $-.726^{* *}$ & $-.709^{* *}$ & $-.577^{* *}$ & $-.638^{* *}$ \\
\hline
\end{tabular}

Nota: $\left({ }^{*}: \mathrm{p}<.05 ;{ }^{* *}: \mathrm{p}<.01 ;\right.$ contraste unilateral)

El segundo objetivo del trabajo era conocer el valor predictivo que la EOMO pudiera tener sobre las diferentes variables medidas. Para ello, se procedió a hacer análisis de regresión donde se tomaba la puntuación en la EOMO como la variable predictora, y considerando, en cada caso, la eficacia lectora, la comprensión lectora, el rendimiento matemático y el rendimiento académico como variables dependientes. 
Tabla 5

Resumen del análisis de regresión con los resultados obtenidos en la EOMO como variable independiente prediciendo la eficacia lectora (TECLE). Organizado por cursos

\begin{tabular}{cccccc}
\hline & B & $\begin{array}{c}\text { Error estándar } \\
\text { de B }\end{array}$ & BETA & $p$ & $R^{2}$ \\
\hline $1^{\mathrm{o}}$ &,- 971 &, 160 &,- 531 & .000 & 0.281 \\
$2^{\mathrm{o}}$ &,- 647 &, 081 &,- 584 & .000 & 0.341 \\
$3^{\mathrm{o}}$ &,- 560 &, 092 &,- 494 & .000 & 0.244 \\
$4^{\mathrm{o}}$ &,- 582 &, 157 &,- 368 & .013 & 0.135 \\
$5^{\mathrm{o}}$ &,,- 827 &, 176 &,- 388 & .002 & 0.151 \\
$6^{\mathrm{o}}$ &,- 397 &, 276 &,- 157 & 0.321 & 0.025 \\
\hline
\end{tabular}

La Tabla 5 muestra que la EOMO es un predictor de la eficacia lectora en todos los cursos, excepto en sexto de primaria. En primero de primaria, da cuenta de un $28 \%$ de la varianza en la eficacia lectora, $F(1,94)=36.82 ; \mathrm{p}<.000$. En segundo de primaria, da cuenta de un $34.1 \%$ de la varianza en la eficacia lectora, con una $F(1,122)=63.09$; $\mathrm{p}<.000$. En tercero de primaria, explica un $24.4 \%$, con una $\mathrm{F}(1,113)=36.79 ; \mathrm{p}<.000$. En cuarto de primaria, da cuenta de un $13.5 \%$ de la varianza en las puntuaciones del TECLE, F $(1,89)=13.78 ; \mathrm{p}<.000$. En quinto da cuenta de un $15.1 \%$ de la varianza en la eficacia lectora, con un $\mathrm{F}(1,121)=22.02 ; \mathrm{p}<.000$. En sexto de primaria, apenas da cuenta de un $2.5 \%$ de la varianza en la eficacia lectora, $F(1,85)=2.07$; $p=.154$. Como se indicaba más arriba, quizá a partir de cierto nivel lector, y no existiendo dificultades graves en lectura, puede que la prueba TECLE no requiera de un procesamiento exigente de la MO, de ahí los datos en este último nivel escolar evaluado.

Tabla 6

Resumen del análisis de regresión con los resultados obtenidos en la EOMO como variable independiente prediciendo la comprensión lectora. Organizado por cursos

\begin{tabular}{lccccc}
\hline & B & $\begin{array}{c}\text { Error estándar } \\
\text { de B }\end{array}$ & BETA & p & R2 \\
\hline $1^{\mathrm{o}}$ & $-3,362$ &, 518 &,- 556 &, 000 & 0.310 \\
$2^{\mathrm{o}}$ & $-1,875$ &, 188 &,- 670 &, 000 & 0.449 \\
$3^{\mathrm{o}}$ & $-1,312$ &, 221 &,- 485 &, 000 & 0.236 \\
$4^{\mathrm{o}}$ & $-1,898$ &, 217 &,- 682 &, 000 & 0.465 \\
$5^{\mathrm{o}}$ & $-1,308$ &, 283 &,- 384 &, 002 & 0.147 \\
$6^{\mathrm{o}}$ & $-1,576$ &, 398 &,- 401 &, 008 & 0.161 \\
\hline
\end{tabular}


En relación con la comprensión lectora, la Tabla 6 muestra que la EOMO es un predictor en todos los cursos, si bien su potencial va decreciendo a medida que se avanza en la etapa. En primero de primaria, explica un 31\% de la varianza en la comprensión lectora, $\mathrm{F}(1,94)=42.14 ; \mathrm{p}<.000$. En segundo de primaria, da cuenta de casi un $45 \%$ de la varianza, con una $\mathrm{F}(1,122)=99.54 ; \mathrm{p}<.000$. En tercero de primaria, explica un $23.6 \%$, con una $\mathrm{F}(1,113)=35.12 ; \mathrm{p}<.000$. En cuarto de primaria, da cuenta de un $46.5 \%$ de la varianza en las puntuaciones del ACL, con una $F(1,89)=76.43 ; \mathrm{p}<.000$. En quinto explica un $14.7 \%$ de la varianza en comprensión lectora, con una $F(1,121)$ $=21.39 ; \mathrm{p}<.000$. En sexto de primaria enuncia un $16.1 \%$ de la varianza en la prueba de comprensión lectora, con una $\mathrm{F}(1,85)=15.71$; $\mathrm{p}<.008$. Como se aprecia, en los dos últimos cursos de la etapa, el valor predictivo de la EOMO decae a un $14 \%$ y un $16 \%$ en quinto y sexto. A este respecto, cabría mencionar la posible influencia de otras variables no tenidas en cuenta en este trabajo, tales como riqueza léxica y gramatical de los textos del ACL, que van aumentando para cada curso. Otra posible variable de influencia podría ser también la presencia de textos con estructura diferente al formato usado frecuentemente en educación (presentación de horarios laborales, tablas de doble entrada), que podrían haber condicionado el rendimiento en comprensión.

La prueba EOMO, tal y como se refleja en la Tabla 7, se muestra como un predictor con respecto al rendimiento matemático en la mayoría de cursos, si bien la varianza explicada es, en general, más baja que con respecto al resto de variables explicadas. En primero de primaria, da cuenta de un $12.1 \%$ de la varianza, $\mathrm{F}(1,94)=13.89 ; \mathrm{p}<.000$. En segundo de primaria, un $17.1 \%$ de la varianza, con una $F(1,122)=25.13 ; p<.000$. En tercero de primaria, a pesar de la significación estadística, apenas explica un $3.3 \%$, con una $\mathrm{F}(1,113)=3.93 ; \mathrm{p}=.05$. En cuarto de primaria, explica un $34.6 \%$ de la varianza en las puntuaciones en cálculo, con una $\mathrm{F}(1,89)=46.65 ; \mathrm{p}<.000$. En quinto explica un $12.2 \%$ de la varianza en la prueba de cálculo, con una $\mathrm{F}(1,121)=17.16 ; \mathrm{p}<.000$. En sexto de primaria da cuenta de un $26.4 \%$ de la varianza, con una $\mathrm{F}(1,85)=29.42 ; \mathrm{p}<.000$. Los resultados en tercero de primaria, junto con la baja correlación con la EOMO, para esta muestra, y con esta prueba utilizada, parecen mostrar que otras variables no tenidas en cuenta tienen relevancia en la ejecución matemática. La consolidación de los algoritmos de la suma y la resta podrían haber condicionado la ejecución en la prueba de cálculo. Igualmente se podría hacer mención a la posibilidad del uso de heurísticos para completar la prueba. Por otro lado, factores motivacionales y/o de procesos atencionales podrían también estar dando cuenta de la baja relación entre la EOMO y el rendimiento matemático.

Tabla 7

Resumen del análisis de regresión con los resultados obtenidos en la EOMO como variable independiente prediciendo el rendimiento matemático. Organizado por cursos

\begin{tabular}{lccccc}
\hline & B & $\begin{array}{c}\text { Error estándar } \\
\text { de B }\end{array}$ & BETA & $p$ & $\boldsymbol{R}^{2}$ \\
\hline $1^{\mathrm{o}}$ &,- 434 &, 116 &,- 359 & 0.012 & 0.129 \\
$2^{\mathrm{o}}$ &,- 254 &, 051 &,- 413 & 0.000 & 0.171 \\
\hline
\end{tabular}




\begin{tabular}{cccccc}
\hline & B & $\begin{array}{c}\text { Error estándar } \\
\text { de B }\end{array}$ & BETA & $p$ & $\boldsymbol{R}^{\mathbf{2}}$ \\
\hline $3^{\mathrm{o}}$ &,- 136 &, 069 &,- 183 & 0.05 & 0.033 \\
$4^{\mathrm{o}}$ &,- 408 &, 060 &,- 589 & 0.000 & 0.346 \\
$5^{\mathrm{o}}$ &,- 382 &, 132 &,- 349 & 0.005 & 0.122 \\
$6^{\mathrm{o}}$ &,- 679 &, 125 &,- 514 & 0.000 & 0.264 \\
\hline
\end{tabular}

Con respecto al rendimiento académico, la Tabla 8 muestra a la EOMO con la mayor capacidad explicativa de varianza. En primero de primaria, revela un $48 \%$ de la varianza, $\mathrm{F}(1,94)=86.61 ; \mathrm{p}<.000$. En segundo de primaria, da cuenta de un $61 \%$ de la varianza, con una $\mathrm{F}(1,122)=190.59 ; \mathrm{p}<.000$. En tercero de primaria explica un $52.8 \%$, con una $\mathrm{F}(1,113)=127.33 ; \mathrm{p}<.000$. En cuarto de primaria, manifiesta un $50.3 \%$ de la varianza en la nota media, con una $\mathrm{F}(1,89)=89.12 ; \mathrm{p}<.000$. En quinto explica un $33.3 \%$ de la varianza en el rendimiento académico, con una $\mathrm{F}(1,121)=61.90 ; \mathrm{p}<.000$. En sexto de primaria revela un $40 \%$ de la varianza de los resultados académicos, con una $\mathrm{F}(1,85)=56.17 ; \mathrm{p}<.000$.

Tabla 8

Resumen del análisis de regresión con los resultados obtenidos en la EOMO como variable independiente prediciendo la nota media. Organizado por cursos

\begin{tabular}{lccccc}
\hline & B & $\begin{array}{c}\text { Error estándar } \\
\text { de B }\end{array}$ & BETA & $p$ & $R^{2}$ \\
\hline $1^{\mathrm{o}}$ & 9.07 & .016 & -.692 & .000 & .480 \\
$2^{\mathrm{o}}$ & 9.26 & .010 & -.781 & .000 & .610 \\
$3^{\mathrm{o}}$ & 8.19 & .013 & -.726 & .000 & .528 \\
$4^{\mathrm{o}}$ & 7.99 & .018 & -.709 & .000 & .503 \\
$5^{\mathrm{o}}$ & 7.67 & .020 & -.577 & .000 & .333 \\
$6^{\mathrm{o}}$ & 8.16 & .032 & -.638 & .000 & .407 \\
\hline
\end{tabular}

\section{Discusión y conclusiones}

Operativizar medidas para la prevención y detección de DA requiere de una inversión amplia de tiempo y recursos, dado que se debe aplicar una gran batería de pruebas a un elevado número de alumnos/as. Contar con instrumentos ágiles en su corrección, así como válidos y fiables, que presenten relaciones con las habilidades instrumentales evaluadas en los procesos de prevención de DA, podría permitir una optimización y mejor eficiencia del proceso. La MO se ha mostrado como un factor cognitivo altamente relacionado con las DA (Raghubar, Barnes y Hecht, 2010; Kudo, Lussier y Swanson, 2015). Sin embargo, hasta ahora, la medición de la MO requería de 
administraciones individuales de pruebas que medían este constructo. En el contexto educativo se necesitan procedimientos ágiles que optimicen los procesos de valoración de la capacidad de MO. La EOMO muestra unas características que se ajustan a las necesidades aquí planteadas.

Los datos obtenidos en este trabajo ponen de manifiesto la relación entre la EOMO y diferentes variables relacionadas con el rendimiento académico, lo que podría apuntar a considerar esta escala como un filtro o screening que permitiera reducir el número de alumnos y alumnas a valorar en los planes de prevención y detección de DA.

Con relación al primer objetivo propuesto, los datos muestran una relación significativa de la EOMO con la eficacia lectora, la comprensión lectora, el rendimiento matemático y el rendimiento académico, a lo largo de toda la etapa de educación primaria. En este sentido, se confirma la primera hipótesis de este trabajo: existe una relación inversa entre las puntuaciones en EOMO y la eficacia lectora, comprensión lectora, rendimiento matemático y rendimiento académico. Hemos de señalar, no obstante, que el TECLE no se muestra "cognitivamente exigente" en alumnos/as de sexto de primaria, no encontrándose relaciones significativas entre éste y la EOMO. Sin embargo, también podríamos plantear, además de los datos de Swanson (2003) citados más arriba, el hecho de que no existieran en la muestra de este nivel educativo alumnos/as con dificultades en lectura. Consideramos añadir a este respecto que esta ausencia de relación entre el TECLE y la EOMO no resulta significativa a efectos prácticos. El TECLE es un instrumento que valora la eficacia lectora, a fin de detectar dificultades específicas de lectura. Pero en consonancia con la normativa planteada en la introducción de este trabajo, y de acuerdo a la literatura científica, es en torno a los cursos de tercero y cuarto cuando se puede establecer un patrón lector alterado como una dificultad específica de lectura. Y en estos niveles, el EOMO y el TECLE sí que muestran una relación significativa. Con respecto a la comprensión lectora, el EOMO muestra relaciones menos intensas a medida que se avanza en la etapa. Como se mencionaba más arriba, quizá factores atribuibles a las propiedades de la prueba en niveles superiores podrían estar dando cuenta de esta tendencia. También se debe señalar la baja relación que la EOMO ha mostrado con el rendimiento matemático en tercero de primaria. Entendemos, en este caso también, que factores atribuibles a la ejecución de la prueba de cálculo pueden estar dando cuenta de la baja relación entre ambas variables, dado que la relación entre EOMO y la nota en Matemáticas es mucho mayor.

Respecto al segundo objetivo, con un valor más intrínseco que propedéutico, los datos han mostrado el carácter ciertamente predictivo de la EOMO sobre todas las variables, confirmándose la segunda hipótesis planteada en este estudio. Debemos señalar, no obstante, y en consonancia con lo anteriormente expuesto, que en el caso del rendimiento matemático en tercero de primaria explica muy poca varianza a pesar de la significación de la relación entre ambas variables. Con respecto al rendimiento matemático, la varianza explicada por la EOMO es, en general, menor en todos los cursos, en comparación con el resto de variables. Por otro lado, es especialmente reseñable el alto valor predictivo que la EOMO muestra sobre el rendimiento académico. La varianza explicada por la EOMO a lo largo de los diferentes cursos oscila entre el $33 \%$ y el 61\%, lo que supone plantear que, para la muestra obtenida, la EOMO explica, 
como mínimo, un tercio de la nota media, llegando a ser responsable de casi dos tercios de la nota media en segundo de primaria.

Debemos señalar también las limitaciones de este trabajo. La obtención de datos en el contexto educativo, desde un enfoque centrado en la intervención, condicionó el diseño del estudio. El diseño cuasi-experimental y el muestreo incidental reducen la potencia estadística. Entendemos que tamaños muestrales mayores, así como muestreos aleatorios habrían dotado de mayor poder estadístico y validez a esta investigación.

También somos conscientes de la moderación con que debemos considerar la EOMO como predictor de las variables estudiadas. Entendemos este trabajo como un precursor a partir del cual ahondar más profundamente en las cualidades de esta escala. El uso de diseños experimentales y muestras amplias podría permitir conocer puntajes críticos en la EOMO a partir de los que considerar la presencia de DA; los datos en este estudio apuntan que, cuanto mayores son las puntuaciones, más dificultades existen, pero no se ha contado con muestras amplias para poder definir puntos normativos.

Este trabajo, en definitiva, apunta a la viabilidad de la EOMO para convertirse en un instrumento de cribado en los procesos de prevención-detección de DA, permitiendo poner el foco en aquel alumnado que muestra signos compatibles con dificultades en $\mathrm{MO}$, las cuales se muestran relacionadas con las DA tal y como se ha demostrado en diversos estudios científicos ya citados a lo largo de este trabajo.

A la luz de los datos, consideramos oportuno continuar esta línea de investigación en coordinación con organismos de la Consejería de Educación, Juventud y Deportes de la Región de Murcia y con el Equipo de Dificultades de Aprendizaje, lo que permitirá contar con muestras mayores, a fin de poder generalizar los resultados, así como el estudio de posibles perfiles diferenciales de la EOMO en función de las DA que presente el alumnado.

\section{References}

Alloway, T. P., Alloway, R. G. y Wootan, S. (2014). Home sweet home: Does where you live matter to working memory and other cognitive skills? Journal of Experimental Child Psychology, 124, 124-131.

Ashkenazi, S., Rosenberg-Lee, M. Metcalfe, A. W. S., Swigart, A. G. y Menon, V. (2013). Visuo- spatial working memory is an important source of domain-general vulnerability in the development of arithmetic cognition. Neuropsychologia, 51(11), 2305-2317. doi: 10.1016/j.neuropsychologia.2013.06.031

Baddeley, A. D. (2003). Working Memory: Looking back and looking forward. Nature Reviews (Neuroscience), 4, 829- 839. doi: 10.1038/nrn1201

Baddeley, A. D. y Hitch, G. J. (1994). Working Memory. En G. H. Bower (Ed.), The Psychology of learning and motivation: Advances in research and theory (Vol. 8, pp. 47- 90). New York: Academic Press.

Blankenship, T. L., O’Neill, M., Ross, A., y Bell, M. A. (2015). Working memory and recollection contribute to academic achievement. Learning and Individual Differences, 43, 164-169. doi.org/10.1016/j.lindif.2015.08.020

Catalá, G., Catalá, M., Molina, E. y Monclús, R. (2001). Evaluación de la comprensión lectora. Barcelona: Grao. 
Decreto 359/2009, de 30 de octubre, por el que se establece y regula la respuesta educativa a la diversidad del alumnado en la Comunidad Autónoma de la Región de Murcia. Boletín Oficial de la Región de Murcia, 254, 3 de noviembre de 2009, pp. 57608-57647.

Durán, M., Veleiro, A., Risso, A. Peralbo, M., García, M. y Brenlla, J. C. (2016) Dificultades en lectura y funcionamiento ejecutivo en $1^{\circ}$ de Educación Primaria. La aportación de Childhood Executive Functioning Inventory. En J.L. Castejón (Coord.), Actas del VIII Congreso Internacional de Psicología y Educación. (p. 197). Alicante: ACIPE- Asociación Científica de Psicología y Educación.

Esquivel, I., Martínez, W., Córdoba, R. y Reyes, C. (2016). Memoria operativa y lectura comprensiva: medición con pruebas de amplitud lectora y tipo cloze en ámbitos pre-universitarios. Apertura, 8(2), 38-53.

Fischbach, A., Könen, T., Rietz, C. S. y Hasselhorn, M. (2014). What is not working in working memory of children with literacy disorders? Evidence from a three-yearlongitudinal study. Reading and Writing, 27(2), 267-286.

Friso-Van den Bos, I., Van der Ven, S. H. G., Kroesbergen, E. H. y Van Luit, J. E. H. (2013). Working memory and mathematics in primary school children: A meta-analysis. Educational Research Review, 19, 29-44.

García-Madruga, J. A. y Fernández; T. (2008). Memoria operativa, comprensión lectora y razonamiento en la educación secundaria. Anuario de Psicología, 39(1), 133-157.

Gathercole, S. E., Woolgar, F., Kievit, R. A., Astle, D., Manly, T y Holmes, J. (2016). How Common are WM Deficits in Children with Difficulties in Reading and Mathematics? Journal of Applied Research in Memory Cognition, 5, 384-394.

Gómez, L., Pérez, M. A., Hernández, L., Martínez, C., Marín, J., Cuello, V. ... Garrido, C. F. (2012). Escala Observacional de Memoria Operativa. En J. Navarro; Ma․ Tª Fernández; F. J. Soto y F. Tortosa (Coords.), Respuestas flexibles en contextos educativos diversos (Vol. 1, pp. 1-6). Murcia: Consejería de Educación, Formación y Empleo.

Gómez-Veiga, I., Vila, J. O., García-Madruga, J. A., Contreras, A. y Elosúa, M. R. (2013). Comprensión lectora y procesos ejecutivos de la memoria operativa. Psicología Educativa, 19, 103-111.

Gutiérrez, F., García-Madruga, J. A., Elosúa, M. R., Luque, J. L. y Gárate, M. (2002). Memoria operativa y comprensión lectora: algunas cuestiones básicas. Acción Psicológica, 1, 45-68.

Gutiérrez-Martínez, F., y Ramos, M. (2014). La memoria operativa como capacidad predictora del rendimiento escolar. Estudio de adaptación de una medida de memoria operativa para niños y adolescentes. Psicología Educativa, 20, 1-10.

Guzmán, B., Véliz, M. y Reyes, F. (2017). Memoria operativa, comprensión lectora y rendimiento escolar. Literatura y Lingüística, 35, 379-404.

Hubber, P. J., Gilmore, C. y Cragg, L. (2014). The roles of the central executive and visuospatial storage in mental arithmetic: a comparison across strategies. Quartely Journal of Experimental Psychology, 67(5), 936-954

Klesczewski, J., Brandenburg, J., Fischbach, A., Schuchardt, K., Grube, D., Hasselhorn, M. y Büttner, G. (2018). Development of Working Memory from Grade 3 to 5: Differences between Children With and Without Mathematical Learning Difficulties. International Journal of Disability, Development and Education 0, 1-17. doi:10.1080/1034 912X.2017.1419555 
Kolkman, M. E., Hoijtink, H. J. A., Kroesbergen, E. H. y Leseman, P. P. M. (2013). The role of executive functions in numerical magnitude skills. Learning and Individual Differences, 24, 145-151.

Kroesbergen, E. H., Noordende, J. E. y Kolkman, M. E. (2014). Training working memory in kindergarten children: effects on working memory early numeracy. Journal of Child Neurology, 20(1), 23-37.

Kudo, M. F., Lussier, C. M. y Swanson, H. L. (2015). Reading disabilities in children: A selective meta-analysis of the cognitive literature. Research in Developmental Disabilities, 40(5), 51-62.

Ley Orgánica 8/2013, de 9 de diciembre, para la mejora de la calidad educativa. Boletín Oficial del Estado, 295, 10 de diciembre de 2013, pp. 97858-97921.

McDonald, P. A. y Berg, D. H. (2017). Identifying the nature of impairments in executive functioning and working memory of children with severe difficulties in arithmetic. Child Neuropsychology, 0, 1-16. doi:10.1080/09297049.2017.1377694

Maehler, C. y Schuchardt, K. (2016). The importance of working memory for school achievement in primary school children with intellectual or learning disabilities. Research in Developmental Disabilities, 58, 1-8.

Marín, J. y Carrillo, M. S. (1999). Test Colectivo de Eficacia Lectora (TECLE). Material no publicado.

Moura, O., Simões, M. R. y Pereira, M. (2015). Working Memory in Portuguese Children With Developmental Dyslexia. Applied Neuropsychology Child, 4(4), 237-248.

Raghubar, K., Barnes, M. A. y Hecht, S. A. (2010). Working memory and mathematics: A review of developmental, individual difference, and cognitive approaches. Learning and Individual Differences, 20(2), 110-122. doi: 10.1016/j.lindif.2009.10.005

Resolución de 17 de diciembre de 2012, de la Dirección General de Planificación y Ordenación Educativa por la que dictan orientaciones para la atención educativa del alumnado que presenta dificultades de aprendizaje. Boletín Oficial de la Región de Murcia, 295, 22 de diciembre de 2012, pp. 51180-51189.

Statistical Package for the Social Sciences (SPSS) (29.0). [Programa Informático]. Nueva York: International Business Machines (IBM).

Swanson, H. L. (2003). Age-related differences in learning disabled and skilled readers working memory. Journal of Experimental Child Psychology, 85(1), 1-31.

Vidal, S. (2012). Procedimiento para el Análisis del Perfil Lector y Detección de Dificultades en Lectura. Comunicación presentada en el I Congreso Nacional de Dificultades de Aprendizaje, 18-20 de octubre, Murcia.

Yuste, C., Martínez, R. y Galve, J. L. (2011). Batería de Aptitudes Diferenciales y Generales Renovada BADYG. Madrid: CEPE.

Fecha de recepción: 4 de diciembre de 2017.

Fecha de revisión: 9 de enero de 2018.

Fecha de aceptación: 8 de abril de 2019 . 
